\title{
Particle Induced Surface Erosion - Tumbling and Direct Impact; a High-speed Electrochemical, Acoustic and Visual study
}

Peter R. Birkin ${ }^{* a}$ and Jennifer L. Barber ${ }^{a}$

${ }^{*}$ Corresponding author

prb2@soton.ac.uk

${ }^{a}$ Chemistry, University of Southampton, Highfield. Southampton, SO171BJ, UK

\begin{abstract}
A technique that monitors the impedance of a $250 \mu \mathrm{m}$ diameter aluminium electrode as a function of time with a $2 \mu \mathrm{s}$ resolution as sand particles are impinged on the solid/liquid interface is reported. The detection of individual particles as they approach an electrode, before any erosion/corrosion was registered, is demonstrated. This study shows that at least two types of erosion mechanisms are possible; direct or 'primary' impact and tumbling or 'scrape' events. The primary impact events are correlated to the acoustic emission from the environment which is shown to be significant for these events, whereas scrape events appear to produce far weaker acoustic emission signatures under the conditions employed. The velocities of the particles are reported and are of the order of 6-8 $\mathrm{m} \mathrm{s}^{-1}$ at the jet mouth. However, high-speed imaging of the particles as they strike the substrate indicates a significant deceleration prior to impact and an order of magnitude reduction in kinetic energy compared to that as it exits the jet.
\end{abstract}

Keywords: Electrochemistry, Solid particle erosion, Impact, Acoustic emission 


\section{Introduction}

Damage to the solid/liquid boundary may have a profound effect on the material and structure in question. This, often parasitic, effect can be generated in a number of ways. Two common examples are cavitation (both flow[1] and through the application of power ultrasound[2,3]) and particle impingement[4]. In the first of these examples, the physical conditions have, under the correct conditions, the ability to generate transient bubbles or voids which collapse and erode the surface. This cavitation process (and the subsequent erosion of the interface[5,6]) is surprisingly diverse and exists in both natural[7,8] and artificial systems[9]. In the second example, liquids in motion which contain particles are also able to cause surface damage[4]. In order to characterise this erosion/corrosion process, many different experimental strategies have been deployed including visual inspection of the interface, simulation of the system[10], measurements of acoustic emission[11], monitoring of resistance changes of a substrate[12], and electrochemical detection[13]. Electrochemical detection of particle impact at an interface has many appealing qualities. The effect of individual particle impacts, for example, can be measured by the detection of erosion/corrosion transients from a passive interface under electrochemical control. Here, the events that cause damage to the interface are detected through the repassivation process and are typically recorded as anodic current time transients. This technique was first developed to investigate cavitation produced by power ultrasound and its erosion/corrosion effects on a passivated interface[5,6]. Complementary techniques can be combined with these electrochemical studies[14]. For example, high-speed imaging and acoustic characterisation can also be useful in characterising an often complex experimental matrix[15]. However, it is always desirable to gather more data from the electrochemical sensor alone. This could include an understanding of those events that cause erosion of the interface and those which impinge on the material in question without the generation of accompanied surface damage. In order to do this a more elaborate approach must be adopted. 
This could include the use of arrays of electrodes[16,17], or as outlined here, the use of a single electrode which is able to sense changes in the local electrochemical environment $[18,19]$. In this later case, the technique must be able to have the spatial and temporal resolution necessary to follow the dynamics of the system under investigation (typically on the $\mu$ s timescale). One such approach would be the use of electrochemical impedance measurements[20]. While changes to the electrochemical environment have been detailed using such an impedance approach in a variety of systems[18,21,22], a study with sufficiently high temporal and spatial resolution to characterise individual particle events has yet to be described. Such an approach, as presented here, would employ high-speed $\mathrm{AC}$ impedance to determine the local environment around the electrode before, during and after a particle impacts onto the surface in question. Although impedance characterisation of an interface (some exposed to cavitation) has been reported previously[23-26] (for lithium interfaces for example[27]), the dynamics of these studies have not been suited to the investigation of individual erosion/corrosion events caused by rapid events (e.g. particle collision with an electrode). However, it has recently been shown that the measurement of impedance of an electrode exposed to cavitation can be performed with a time resolution of $2 \mu \mathrm{s}[28]$. This study showed that the growth and collapse of cavitation bubbles, produced by continuous ultrasound, could be followed prior to any conventional electrochemical signal (e.g. the corrosion process which occurs after bubble collapse) being registered. Clearly, considering the similarities between cavitation damage and particle impact, this AC impedance technique would also be suited to the study of particle impact[29-34] onto an electrode. In order to function, the high-speed impedance method employed relies on the imposition of a suitable AC perturbation to the potential of the electrode interface. This perturbation, combined with the current response of the electrode can be used to extract the electrochemical impedance of the electrode as a function of time. In the absence of any perturbing phenomena[23,35,36] (whether that be a bubble, particle or surface related for 
example) the impedance of the electrode should be constant. However, bubbles and particles perturb the environment around the electrode causing changes in the impedance detected. This change in impedance can be evaluated along with data indicating the Faradaic current that is generated. Critically, the presence of the particles can be detected locally before the impact event occurs and in the absence of an erosion/corrosion event. This will give key information on the position of the particle prior to the data gathered from conventional electrochemical Faradaic currents which show the impact as a subsequent erosion/corrosion event. Lastly the particle impacts are monitored simultaneously with acoustic emission measurements and highspeed imaging. This set of complimentary techniques allows key information on the processes that take place before, during and after individual particle impact onto the solid/liquid interface.

\section{Materials and Methods}

The jet system was constructed from a pump connected to a $\sim 1 \mathrm{~mm}$ diameter glass orifice. Fluid (here an electrolyte) was pumped through the system at velocities in the range of 6-8 $\mathrm{m}$ $\mathrm{s}^{-1}$ (determined by measuring the mass of fluid ejected from the jet over a fixed time or by monitoring the particle velocity within the jet with a high-speed camera). The jet was directed at an electrode substrate which consisted of an aluminium electrode ( $250 \mu \mathrm{m}$ diameter $)$ made by sealing a microwire (Advent Research Materials) in an epoxy resin (Epofix, Struers) filled cuvette on the end of a stainless-steel tube (6 mm OD) - see SI. This tube acted as the counter/reference electrode and a support body for the working electrode while the resin filled cuvette allows the electrode to be detected for imaging from the side (see SI material for more detail). The electrode was polished to a mirror like finish $\left(0.3 \mu \mathrm{m} \mathrm{Al}_{2} \mathrm{O}_{3}\right.$ on Buehler microcloth) prior to experiments. The electrode assembly and hydrophone where then positioned (using micrometers and stages, Time and Precision) with respect to the jet which was fixed. Figure 1 shows a schematic of the experimental setup deployed. 


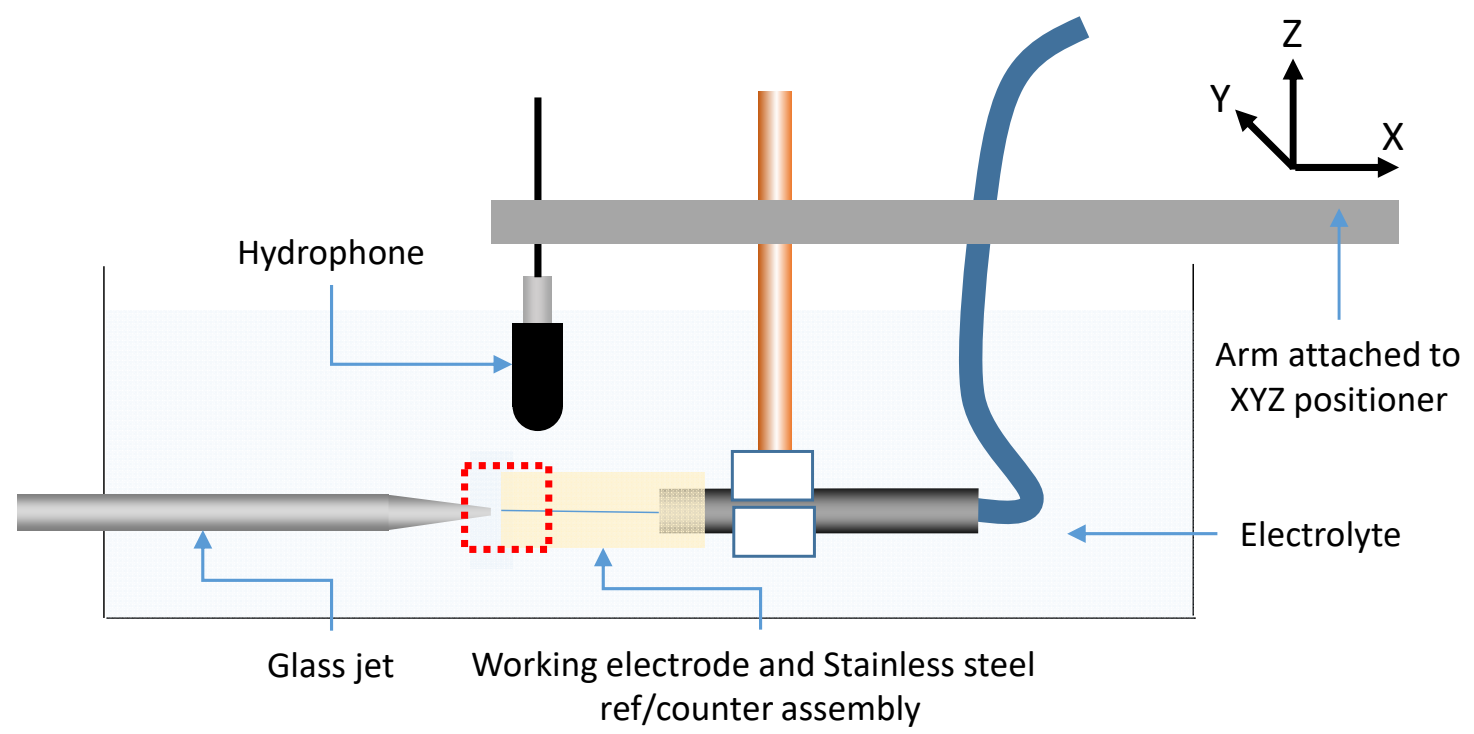

Figure 1. Image showing a representation of the experimental setup used to study sand particle impact onto a $250 \mu \mathrm{m}$ diameter Al electrode. The dotted square shows the region imaged in the high-speed camera experiments.

The pump (Hailea HX8810, not shown in Figure 1) took electrolyte from the main reservoir $\left(\sim 2.5 \mathrm{dm}^{3}\right)$ and pumped it through the jet after passing a loading port where sand was added to the flow system. The high-speed impedance system has been described in detail elsewhere[28]. However, in the experimental setup deployed here and with the additional data requirements needed (the trigger signal in this case), the impedance (and not the uncompensated resistance and effective electrode capacitance) is reported as a function of time. However, in the experiments reported here the impedance of the electrode was determined by recording the data using a Handyscope HS3 (TiePie) and then post processing using a DSP approach (see SI). The aluminium electrode was aligned to the centre of the jet employed (in the YZ plane) and the $\mathrm{X}$ position was controlled and verified from the imaging experiments. The potential of the working electrode was maintained at $0 \mathrm{~V}$ vs. the steel reference. An AC perturbation (a $500 \mathrm{kHz}$ sinusoid, $100 \mathrm{mV}$ zero-to-peak amplitude) was applied using a function generator (Handyscope HS3, TiePie). A calibrated hydrophone (Reson TC4013) was positioned $\sim 1.5 \mathrm{~cm}$ from the tip of the electrode assembly (mounted on the same positioning arm, see Figure 1). The output of the hydrophone was conditioned through an Ortec Brookdeal precision AC 
amplifier (type 9452) and recorded on a Tektronix 2014 scope. An amplification of $62 \mathrm{~dB}$ with band pass frequency limits of $100 \mathrm{~Hz}$ to $1 \mathrm{MHz}$ were applied to the hydrophone signal. The sound pressure was calculated from this amplification and the hydrophone sensitivity, -211.5 $\mathrm{dB}$ re $1 \mathrm{~V} / \mu \mathrm{Pa}$ at $100 \mathrm{kHz}$ (determined by the data sheet supplied with the hydrophone). Timing between the recording devices and the camera was achieved by employing a TTL trigger (see legends for level employed). The complete experimental arrangement it shown as a schematic in the SI data. A Photron APX-RS camera with a Navitar x12 lens was used to image the motion and impact of the particles onto the electrode assembly. The image was backlit using an LED lamp driven by a DC power supply. All solutions consisted of $0.1 \mathrm{~mol} \mathrm{dm}^{-3} \mathrm{Na}_{2} \mathrm{SO}_{4}$ in aerobic purified water $\left(20-25^{\circ} \mathrm{C}\right)$.

\section{Results and Discussion}

Figure 2 shows the velocity of a set of sand particles as they impacted onto the electrode assembly (note ' 0 ' on the $\mathrm{x}$ scale represents the surface of the substrate). It is clear from this data that the velocity of the particles as they leave the jet are on the order of $7 \mathrm{~m} \mathrm{~s}^{-1}$ under these conditions. This velocity remains approximately constant until the particles where within $\sim 250$ $\mu \mathrm{m}$ of the surface; at distances less than this, a significant deceleration was observed. This is significant as these observations suggest that particle impact energy should not be related directly to the jet velocity as this decelerate significantly reduces the particle impact energy compared to the case at the jet exit. For example, consider the case of the particle reported here; a $\sim 300 \mu \mathrm{m}$ diameter approximately spherical sand particle. Under these condition the particle has the kinetic of the order of $0.76 \mu \mathrm{J}$ at the jet mouth while the energy drops to $\sim 16$ $\mathrm{nJ}$ at the surface itself. 


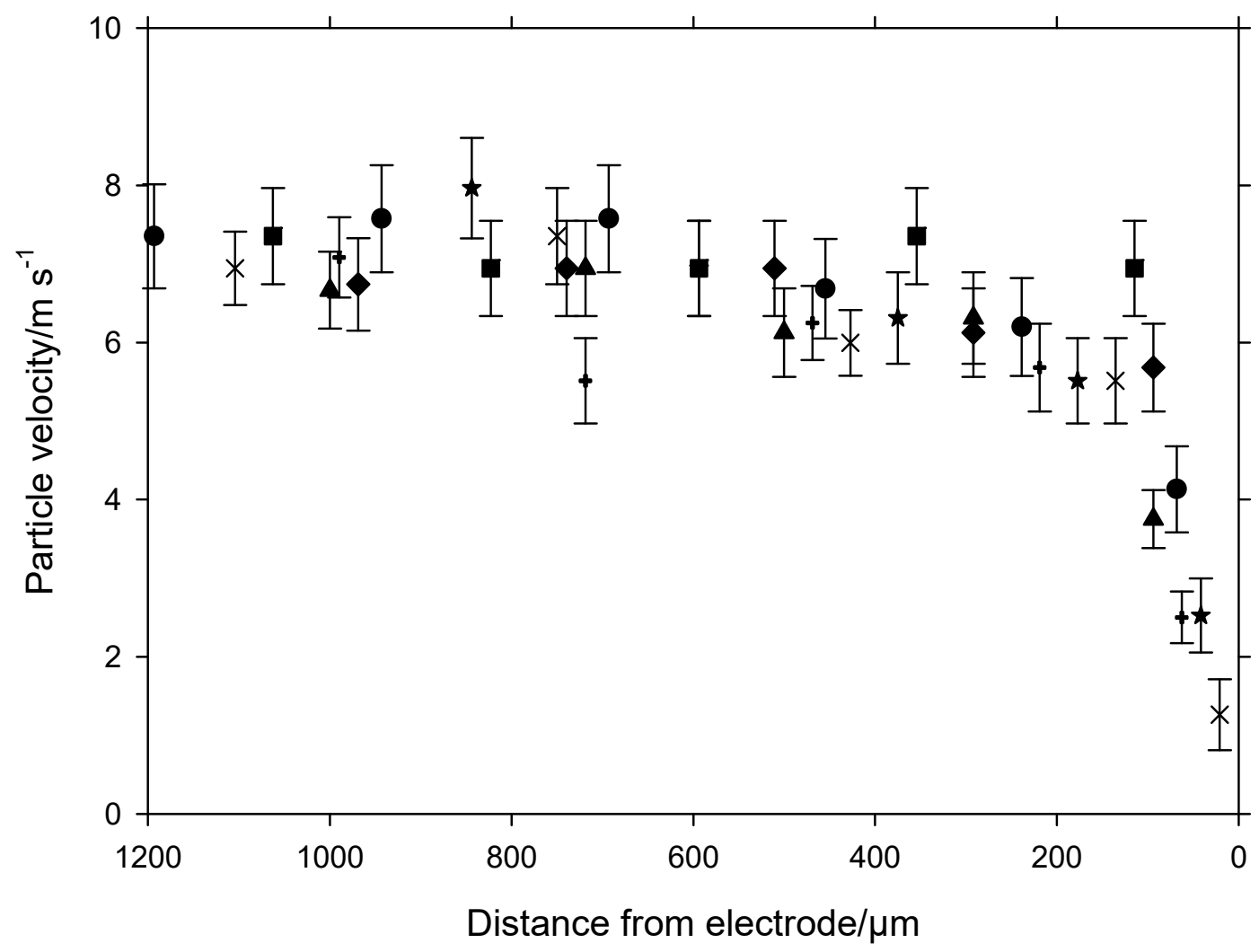

Figure 2. Plot of particle velocity as a function of distance (from the electrode surface) for the particle impacts. The particle impacts occur at $0 \mu \mathrm{m}$. The velocity was calculated for several time intervals between the particles exiting the jet and hitting the electrode surface. The error associated with these values arose from measuring the distances using the positional cursers in Photron FASTCAM Viewer.

Clearly this particle velocity reduction and the corresponding impact energy decrease needs to be considered in studies of this type where the minimum impact energy required to depassivate a surface is estimated. This particle deceleration is also supported by simulations of impinging jets[33,37]. 
(a)

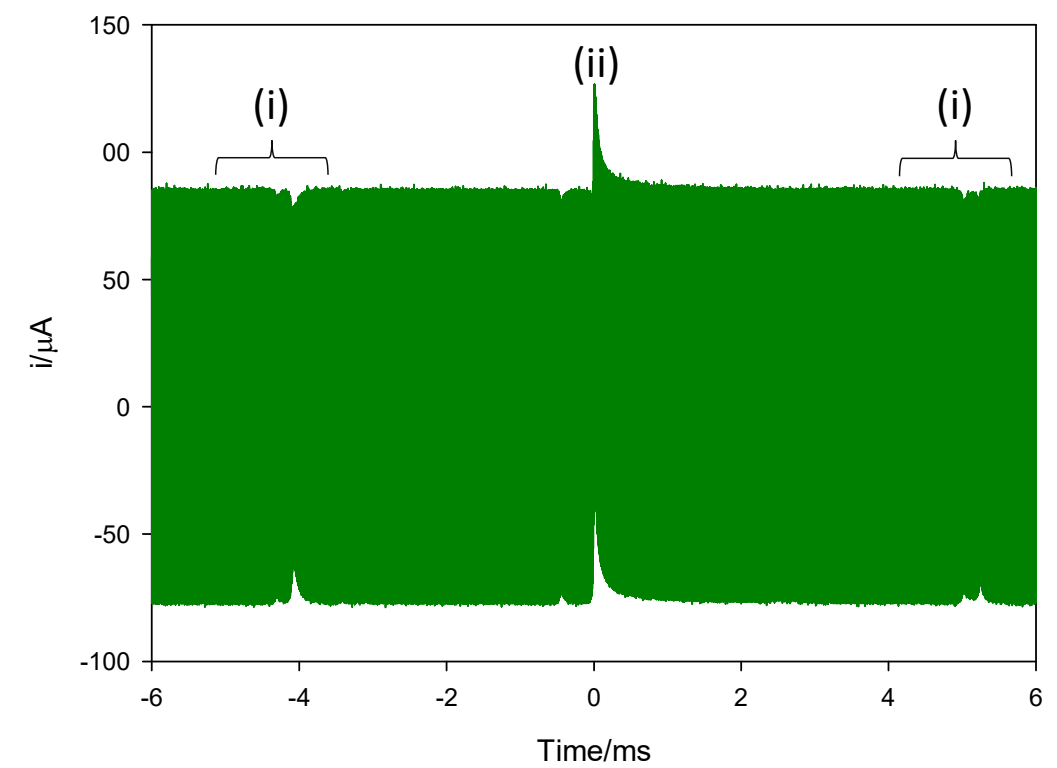

(b)

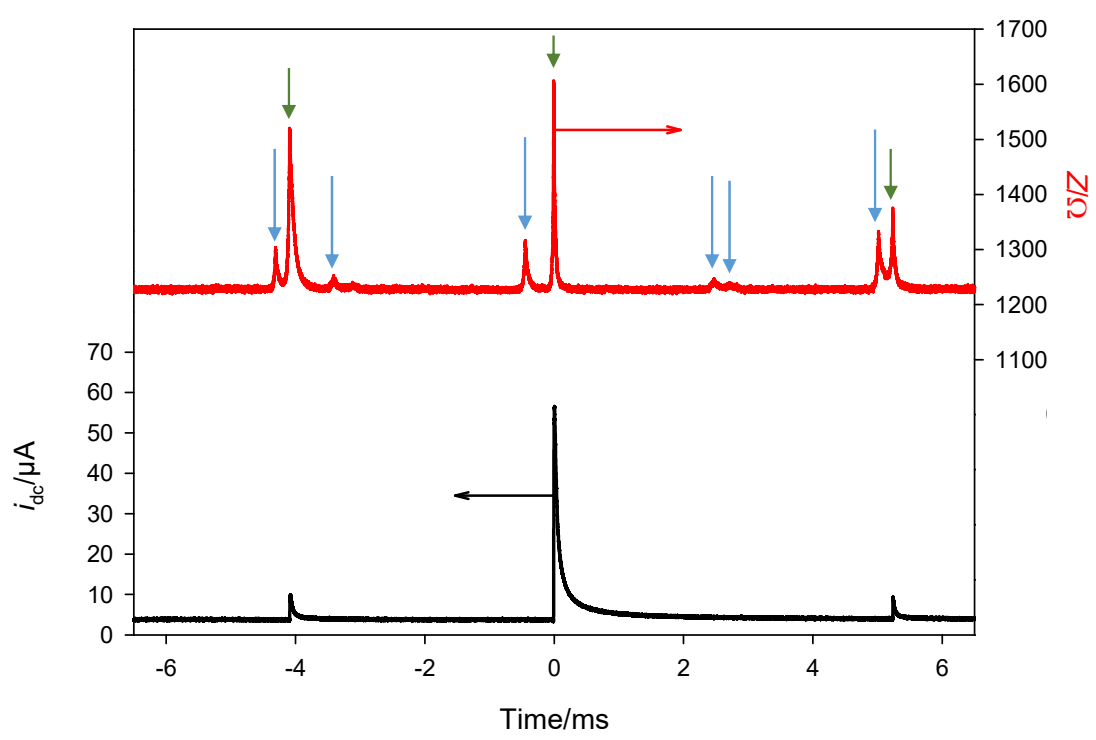

Figure 3. (a) Plot showing the current time (-) recorded for a $250 \mu \mathrm{m}$ diameter aluminium electrode exposed to a jet containing sand particles. The trigger level was set at $110 \mu \mathrm{A}$. The electrode-to-jet separation was $\sim 1 \mathrm{~mm}$. Two types of particle/electrode interaction are noted ((i) and (ii)). (b) Plot showing the impedance $(Z,-)$ and Faradaic current $\left(i_{\mathrm{dc}},-\right)$ as a function of time determined by processing the date shown in (a). The vertical arrows show the effect on the impedance of the electrode of sand particles as they impinge on the electrochemical environment of the electrode. The blue arrows $(\downarrow)$ show events where the aluminium electrode surface was not damaged by the impact while the green arrows $(\downarrow)$ show events where damage was detected.

In order to characterise the impacts of the particle before, during and after impact onto the surface of an electrode, a high-speed electrochemical impedance technique was employed[28]. 
Here an AC perturbation was applied to the electrode and the current response monitored as a function of time while the jet, containing, sand particles, was directed at the electrode surface. Figure 3 shows a set of experimental data and the corresponding analysis obtained from such an experimental approach. Figure 3(a) shows the current time history while figure 3(b) show the extracted data after the DSP approach has been performed. Figure 3(a) shows a broad signal from the sinusoidal voltage perturbation imposed on the electrode. However, there are several notable perturbations on the signal (see annotation and figure 3 legend). These include reductions in amplitude (see figure 3(a), (i) and (ii)) and a significant transient signal above the normal level (see figure 3(a), (ii)). The origins of these perturbations are attributed to the ingress of the sand particles into the electrochemical environment around the electrode and the erosion/corrosion phenomena that can be driven by particle impact. Figure 3(b) shows the impedance and the Faradaic current determined from the data shown in figure 3(a). Here the impedance of the electrode was $\sim 1230 \Omega$ in the absence of particle effects. However, there are several increases in impedance as sand particles, driven by the jet, come close to the electrode surface (see figure 3(b) for example). In addition, the Faradaic current shows three discernible current time transients with the highest recorded at $0 \mathrm{~ms}$ (note the trigger apparatus was set to detect larger transients in the current time signal). These transients in the Faradaic signal are attributed to the collision of the sand particles with the aluminium electrode surface. This collision is of sufficient energy to cause erosion of the passive layer on the electrode surface.

The repassivation of the surface is then detected as a transient anodic current time signal lasting 100's $\mu \mathrm{s}$ in duration. This transient Faradaic process is similar to the effect of inertial cavitation bubbles at electrode surfaces[5,6,38] and has also been reported for particle/electrode impacts[11,39]. However, the data shown in figure 3(b) also shows the influence of the particle on the electrochemical impedance of the electrode in the absence of an erosion/corrosion event. In this case as the particle approached the electrode, its effect on 
the electrochemical atmosphere is recorded in an increase in the impedance of the system (as the insulating nature of the sand particle will increase the uncompensated resistance of the electrode, for example). In the case of figure 3 (b) there are nine impedance transients detected in the time window recorded, three of these electrode/particle encounters produce discernible erosion of the passive layer and are accompanied by a current time transient in the $i_{\mathrm{dc}}$ data. To investigate these interactions further, an additional set of experiments were performed using a high-speed camera and a hydrophone in combination with the $\mathrm{AC}$ impedance technique. In this case, all the experimental data was recorded simultaneously with a common trigger (see SI).

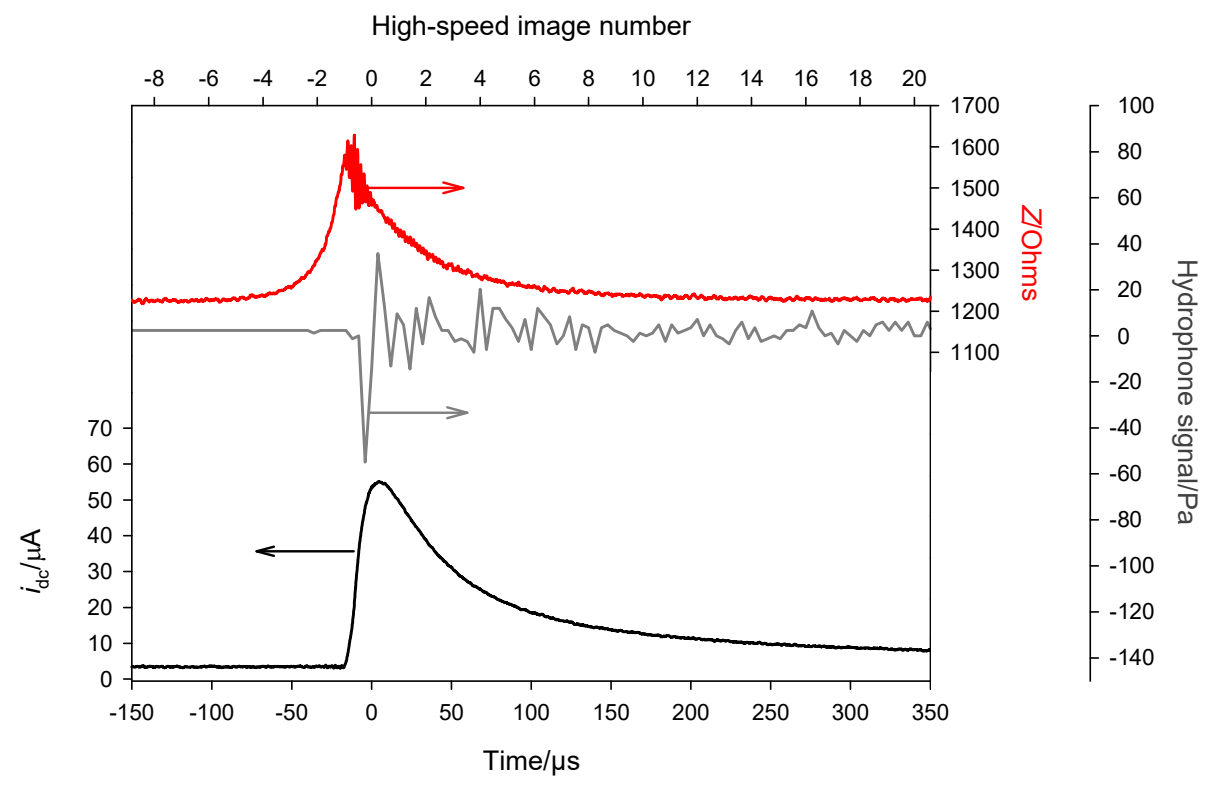

Figure 4. Current time (-), impedance time (-) and acoustic emission $(-)$ traces of events recorded for a $250 \mu \mathrm{m}$ diameter aluminium electrode exposed to a jet of sand particles. The electrode-to-jet separation was $\sim 1 \mathrm{~mm}$. The electrode to hydrophone separation was $\sim 15 \mathrm{~mm}$ (13 $\mathrm{mm}$ from a speed of sound calculation).

Figure 4 shows an example of the Faradaic signal, $i_{\mathrm{dc}}$ and electrochemical impedance $(Z)$ of the aluminium electrode as an individual particle approaches, causes surface erosion and then leaves the interface. The impedance of the electrode shows that the particle was detected as an increase in the electrode impedance $\sim 100 \mu$ s before the Faradaic current at the aluminium electrode was detected (note this transient in the Faradaic signal is thought to be responsible 
for the slight instability manifested on the impedance data). In addition, a significant acoustic transient was detected as the particle hits the surface of the electrode (note there will be a slight delay between the particle impact and the detection of the acoustic signal due to the finite speed of sound in the media; here under the conditions employed this delay time is estimated to be $\sim 10 \mu \mathrm{s}$ ). The impact (of the particle responsible for the data shown in figure 4) with the surface was also recorded with a high-speed camera. Figure 5 shows the individual sand particle responsible for the surface erosion of the electrode as it travels through the liquid jet, decelerates, hits the surface and then moves away from the interface (note the frame number is also shown on figure 4 for completeness). Figure 5 shows that the sand particle in this case was $\sim 300 \mu \mathrm{m}$ in diameter. The asymmetry in the impedance data shown in figure 4 agrees with the images as the particle's velocity after the impact is significantly perturbed as expected by the impact with the surface and can be seen to reside close to the surface of the electrode for a further 1-2 ms. While these single impact events are often observed, other encounters between the particle and the electrode can also be detected. Figure 6 shows an interesting impact where the erosion of the electrode and the acoustic emission were not observed to occur concomitantly. The impedance data in this case shows two transients in close temporal proximity to one another. 


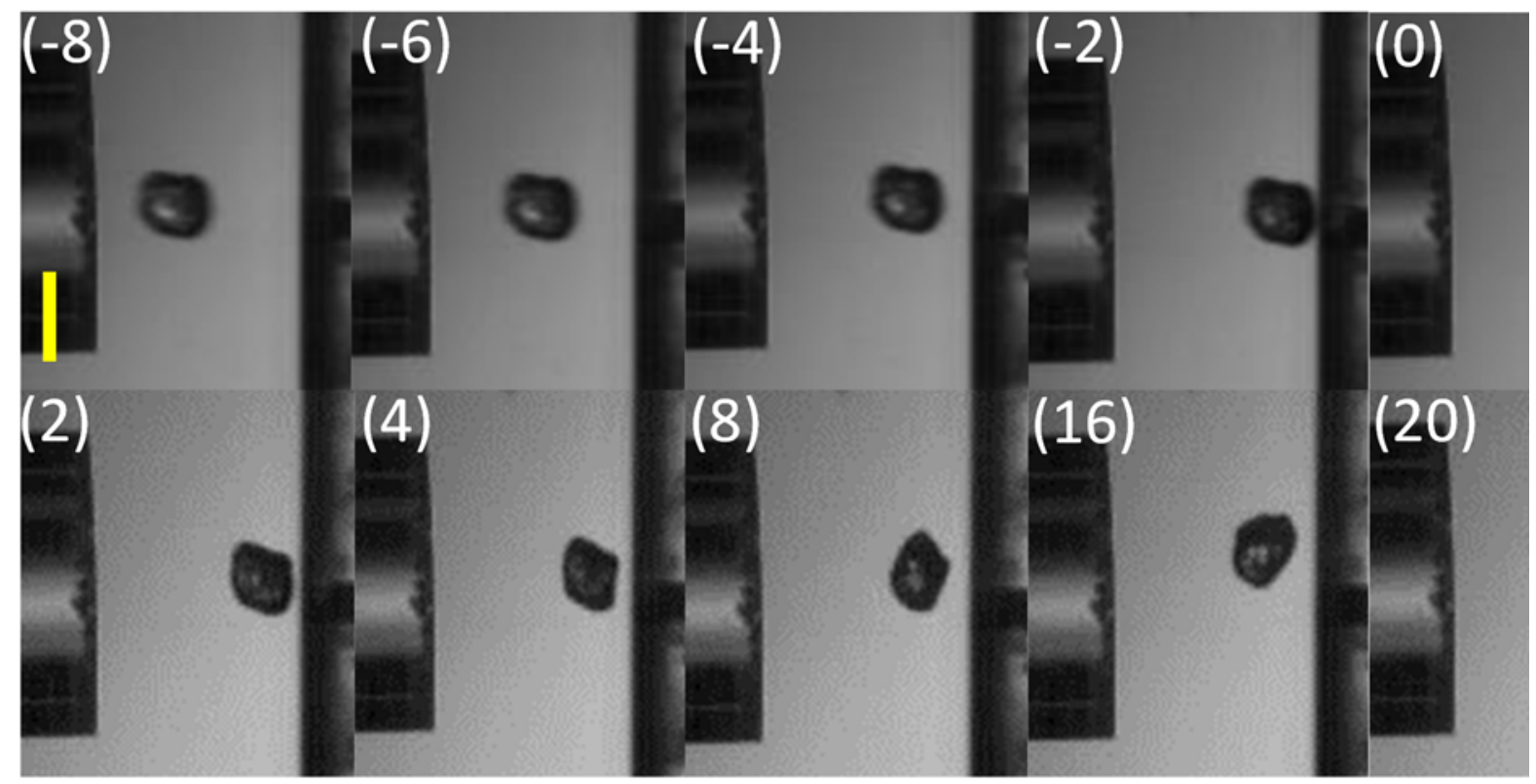

Figure 5. Images from high-Speed camera showing a single sand particle as it approaches the electrode, impacts and then moves away from the surface (see figure 4 shows the impedance, $Z$, and faradaic current, $i_{\mathrm{dc}}$, accompanying these images). The images were recorded at $60000 \mathrm{fps}$ with a 1/60000 s exposure. All other experimental conditions as reported in figure 4 . Image number " 0 " corresponds to the trigger point of the data shown in figure 4. The numbers in parenthesis refer to the image numbers hence -8 corresponds to $133.3 \mu$ s before impact. The scale bar represents $500 \mu \mathrm{m}$.

The first impedance transient (see figure 6 (a)) coincides with a strong acoustic emission while the second (see figure 6 (b)) corresponds to the large erosion/corrosion transient detected by the electrode (centred at $0 \mathrm{~s}$ ). The absence of a significant acoustic transient at the point where the erosion of the surface occurred suggests that the mechanism responsible does not involve direct impact of the particle on the surface of the electrode (as in the case shown in figure 5). 


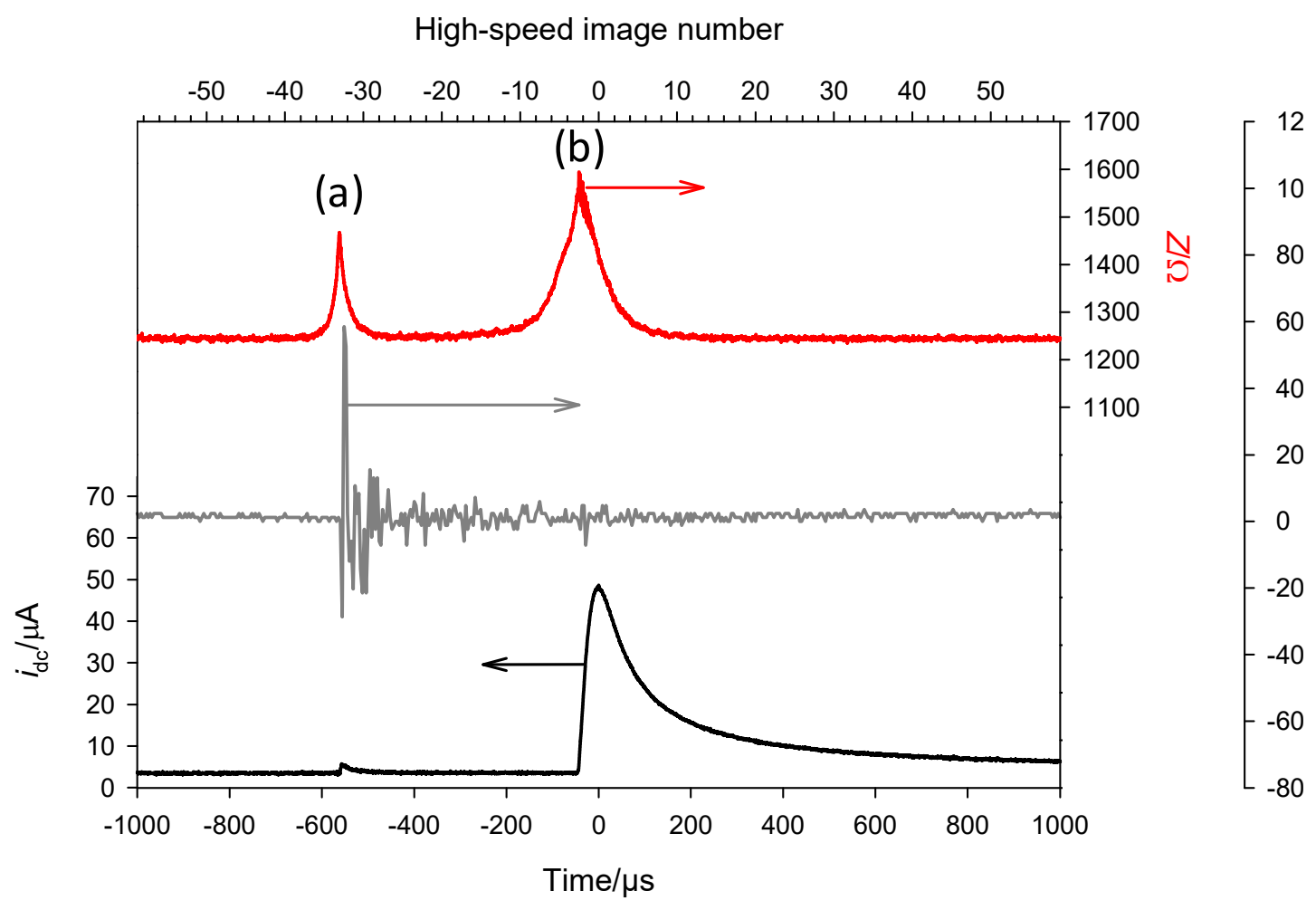

Figure 6. Current time (-), impedance time (-) and acoustic emission $(-)$ traces of events recorded for a $250 \mu \mathrm{m}$ diameter aluminium electrode exposed to a jet of sand particles. Two impacts by the same particle were recorded in the period shown: (a) "primary impact" at $-0.55 \mathrm{~ms}$ and a "scrape" (b) at $0 \mathrm{~ms}$. The electrode-to-jet separation was $\sim 1 \mathrm{~mm}$. The electrode to hydrophone separation was $\sim 15 \mathrm{~mm}$ (13 $\mathrm{mm}$ from a speed of sound calculation).

The high-speed imaging of the electrode/particle encounter elucidates the mechanisms occurring in this case. Figure 7 shows imaging of the particle and its motion with respect to the electrode surface and jet. Initially the particle travels out of the jet and through the space between the jet and the electrode substrate. Deceleration and impact occurs at -550 $\mu$ s in the impedance time history shown. 


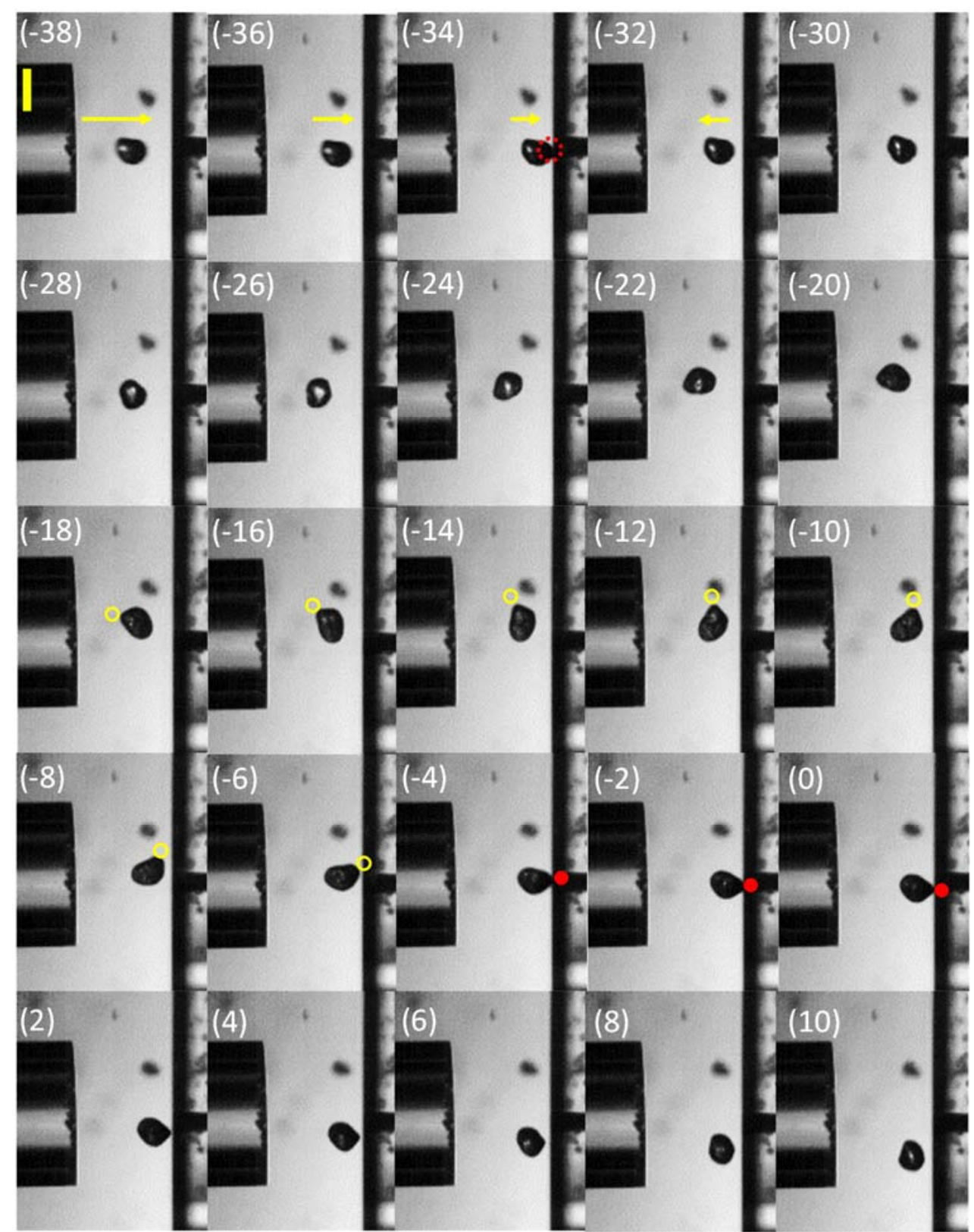

Figure 7. High-Speed Camera images of a single sand particle hitting the surface to cause the peaks $-0.58 \mathrm{~ms}$ and $0 \mathrm{~ms}$ in figure 6 . The images were recorded at 60000 fps $(1 / 60000$ shutter). The frame number w.r.t. the trigger is shown in parenthesis. The arrows show the approach (and velocity) are indicated in the first row. The hollow red dashed circle highlights the first impact, the yellow circle shows the motion of the edge that causes the erosion which occurs in the frames highlighted by a red solid circle. Scale bar (top left) represents $500 \mu \mathrm{m}$.

Figure 7 shows the impact of the particle at frame $-34(-567 \mu$ s) with respect to the trigger.

This impact is accompanied by a significant acoustic event but comparatively minor erosion/corrosion. This is presumably because the exact point of contact of the particle with the surface misses the aluminium interface in this case (an assumption supported by the small 
impedance increase w.r.t figure 6(b)). The particle is then seen to rotate in the fluid in front of the electrode substrate over the next $500 \mu$ s and then scrape the surface. It is this second action that is registered as a second (larger) impedance transient and accompanied with a large erosion/corrosion signal. However, this 'scraping' motion (akin to scratching the electrode an causing erosion/corroision[40,41]), due to the particle rotation that occurs in this case, does not appear to generate a significant acoustic event. This suggests that these type of event, although causing a significant amount of surface damage (note the size of the Faradaic repassivation transient) cannot easily be detected using the acoustic analysis alone. The high-speed images allow for an estimate of the rotational period of the particle $(\sim 500 \mu \mathrm{s}$, estimated from first impact in frame -34 to 'scrape' in frame 4) to be gathered. This corresponds to a rotational energy[42] of $\sim 22 \mathrm{~nJ}$ assuming a spherical geometry with a diameter of $300 \mu \mathrm{m}$. This is approximately the magnitude of the actual direct impact energy (considering the deceleration of the particles as they approach the solid/liquid interface of the electrode substrate). However, the rotation of the particle was not halted by the scrape across the electrode. Hence, we should expect that only a fraction of the rotational energy was required to cause the surface damage registered as the anodic transient centred at $0 \mathrm{~ms}$ in figure 6. The impedance data, in combination with the high-speed imaging and the acoustic data can allow a complete picture of the interaction of individual particles with a surface.

While the direct impact and the rotational 'scrape' events can clearly both cause damage to the interface, it is of interest to consider the likely frequency of both type of events. To this end, a series of experiments were performed where sand particles (and their impact and interaction) were monitored using the $\mathrm{AC}$ technique in combination with high-speed imaging. Over a series of 31 repeats runs (each representing a data set corresponding to $\sim 13 \mathrm{~ms}$ in length) detailing $406 \mathrm{~ms}$ in total time, a significant number (156) of events where analysed. The results of these experiments are summarised in Table 1. 


\begin{tabular}{|l|c|c|c|c|}
\hline & $\begin{array}{c}\text { Primary } \\
\text { events }\end{array}$ & $\begin{array}{c}\text { Rebound } \\
\text { events }\end{array}$ & Scrapes & Total \\
\hline $\begin{array}{l}\text { Impact type from high- } \\
\text { speed imaging }\end{array}$ & 112 & 22 & 21 & 156 \\
\hline Impedance events detected & $51.8 \%$ & $14.1 \%$ & $13.5 \%$ & $100.0 \%$ \\
\hline $\begin{array}{l}\text { Significant Erosion/corrosion event } \\
\text { detected }\end{array}$ & $25.6 \%$ & $0.0 \%$ & $0.6 \%$ & 10 \\
\hline
\end{tabular}

Table1. Distribution of impact events for $406 \mathrm{~ms}$ of electrochemical data combined with high-speed video. The $\%$ in each cell refers to the total number of electrode substrate/particle encounters (156) analysed in the complete data set. Primary events are when the particle leaves the jet and hits the substrate directly, rebound events are when the particle bounces on the surface but appears to then re-hit the interface and scrape events are when the particle was observed to rotate in the space in front of the electrode assembly.

Over the data set analysed three different types of impact event were noted. These were classed as primary impact (see figure 4), rebound (when the particle bounces off the surface and then re-hits the solid substrate surface but without rotation) or finally scrape where after the initial primary impact the particle then goes on to rotate and scrape the electrode assembly surface (see figure 7, for example). Included in these data is a number of particles/electrode encounters that were detected as an impedance transient but did not cause surface erosion. These are attributed to 'near misses' of the electrode surface itself or where there was insufficient impact energy to cause erosion. Almost all surface erosion events were found to be as a result of direct impact of a particle onto the electrode surface. No erosion events were detected during a second impact (rebound hits) suggesting the energies in these cases was insufficient to cause damage. Only one example of an erosion event caused by a tumbling event, or "scrape", was recorded in this data set. This suggests that this type of rotation event is relatively rare compared to the direct event within this experimental setup and with the particles deployed in the system. 


\section{Conclusions}

The motion of individual sand particles within a jet system can be monitored with a highspeed (with a $2 \mu$ s time resolution) AC impedance technique. Particles can be detected as they approach the electrode surface as transient impedance increase before any erosion/corrosion event can be detected. Faradaic transients, caused by the repassivation of the surface damaged by the particle, can also be extracted from the data. High-speed imaging indicates significant deceleration of the particles (and hence a large decrease in kinetic energy) as they approached the solid/liquid interface. Erosion of the surface is caused, in most of the cases recorded, by direct primary impact, however, rotation and 'scraping' events are possible but appear less frequent for the conditions employed.

\section{Acknowledgements}

The authors would like to thank the University of Southampton for support and funding for JLB and the EPSRC (EP/D05849X/1) for funding related to equipment used in the work presented.

\section{References}

[1] S. Hattori, B. Sun, F.. Hammitt, T. Okada, An Application of Bubble Collapse Pulse Height Spectra to Venturi Cavitation Erosion of 1100-O Aluminium, Wear. 103 (1985) $119-131$.

[2] Y.K. Zhou, F.G. Hammitt, Vibratory cavitation erosion in aqueous solutions, Wear. 87 (1983) 163-171. doi:10.1016/0043-1648(83)90031-5.

[3] K. Kikuchi, F.G. Hammitt, Effect of Separation Distance on Cavitation Erosion of 
Vibratory and Stationary Specimens in a Vibratory Facility, Wear. 102 (1985) 211-225.

[4] A.W. Hassel, A.J. Smith, Single particle impact experiments for studying particle induced flow corrosion, Corros. Sci. $49 \quad$ (2007) 231-239. doi:10.1016/j.corsci.2006.05.021.

[5] P.R. Birkin, R. O’Connor, C. Rapple, S.S. Martinez, Electrochemical measurement of erosion from individual cavitation events generated from continuous ultrasound, J. Chem. Soc. Faraday Trans. 94 (1998) 3365-3371.

[6] S. Silva-Martinez, Applications of Ultrasound in Electrochemistry, University of Southampton, 1997.

[7] S.N. Patek, R.L. Korff, R.L. Caldwell, Deadly strike mechanism of a mantis shrimp, Nature. 428 (2004) 819-820.

[8] S.N. Patek, R.L. Caldwell, Extreme impact and cavitation forces of a biological hammer: strike forces of the peacock mantis shrimp Odontodactylus scyllarus., J. Exp. Biol. 208 (2005) 3655-64. doi:10.1242/jeb.01831.

[9] F.R. Young, Cavitation, Imperial College Press, London, 1999.

[10] H.S. Grewal, H. Singh, E.S. Yoon, Interplay between erodent concentration and impingement angle for erosion in dilute water-sand flows, Wear. 332-333 (2015) 11111119. doi:10.1016/j.wear.2015.02.039.

[11] G.. Burstein, K. Sasaki, Detecting electrochemical transients generated by erosioncorrosion, Electrochim. Acta. 46 (2001) 3675-3683. doi:10.1016/S00134686(01)00646-6. 
[12] R.E. Vieira, M. Parsi, P. Zahedi, B.S. McLaury, S.A. Shirazi, Electrical resistance probe measurements of solid particle erosion in multiphase annular flow, Wear. 382-383 (2017) 15-28. doi:10.1016/j.wear.2017.04.005.

[13] K. Sasaki, G.T. Burnstein, Detecting electrochemical transients generated by erosioncorrosion, 46 (2001) 3675-3683.

[14] P.R. Birkin, T.G. Leighton, D.G. Offin, C.J.B. Vian, Towards the understanding of a complex cavitation environment - electrochemistry, acoustics and imaging observations, (n.d.) 1-9.

[15] P.R. Birkin, D.G. Offin, C.J.B. Vian, T.G. Leighton, Multiple observations of cavitation cluster dynamics close to an ultrasonic horn tip., J. Acoust. Soc. Am. 130 (2011) 337988. doi:10.1121/1.3650536.

[16] E. Maisonhaute, B. a. Brookes, R.G. Compton, Surface Acoustic Cavitation Understood via Nanosecond Electrochemistry. 2. The Motion of Acoustic Bubbles, J. Phys. Chem. B. 106 (2002) 3166-3172. doi:10.1021/jp013448a.

[17] E. Maisonhaute, P.C. White, R.G. Compton, Surface acoustic cavitation understood by nanosecond electrochemistry, J. Phys. Chem. B. 105 (2001) 12087-12091.

[18] C. Gabrielli, Real-Time Measurement of Electrolyte Resistance Fluctuations, J. Electrochem. Soc. 138 (1991) L82. doi:10.1149/1.2085517.

[19] C. Gabrielli, F. Huet, R.P. Nogueira, Electrochemical Noise Measurements of Coalescence and Gas-Oscillator Phenomena on Gas-Evolving Electrodes, J. Electrochem. Soc. 149 (2002) E71-E77. doi:10.1149/1.1445432.

[20] M.E. Orazem, B. Tribollet, Electrochemical Impedance Spectroscopy, Wiley, New 
Jersey, 2008.

[21] S.C. Case, Coalescence of low-viscosity fluids in air, Phys. Rev. E - Stat. Nonlinear, Soft Matter Phys. 79 (2009) 1-10. doi:10.1103/PhysRevE.79.026307.

[22] S.C. Case, S.R. Nagel, Coalescence in low-viscosity liquids, Phys. Rev. Lett. 100 (2008) 1-4. doi:10.1103/PhysRevLett.100.084503.

[23] H. Bouazaze, J. Fransaer, F. Huet, P. Rousseau, V. Vivier, Electrolyte-resistance change due to an insulating sphere in contact with a disk electrode, Electrochim. Acta. 55 (2010) 1645-1655. doi:10.1016/j.electacta.2009.10.043.

[24] H. Bouazaze, F. Huet, R.P. Nogueira, A new approach for monitoring corrosion and flow characteristics in oil/brine mixtures, Electrochim. Acta. 50 (2005) 2081-2090. doi:10.1016/j.electacta.2004.08.051.

[25] J. Ryl, J. Wysocka, P. Slepski, K. Darowicki, Instantaneous impedance monitoring of synergistic effect between cavitation erosion and corrosion processes, Electrochim. Acta. 203 (2015) 388-395. doi:10.1016/j.electacta.2016.01.216.

[26] G.A. Zhang, L.Y. Xu, Y.F. Cheng, Mechanistic aspects of electrochemical corrosion of aluminum alloy in ethylene glycol-water solution, Electrochim. Acta. 53 (2008) 82458252. doi:10.1016/j.electacta.2008.06.043.

[27] P.R. Birkin, E. Eweka, J.R. Owen, The effect of ultrasound on lithium surfaces in propylene carbonate, J. Power Sources. 81-82 (1999).

[28] P.R. Birkin, T.M. Foley, J.L. Barber, H.L. Martin, Microsecond resolution of cavitation bubble dynamics using a high-speed electrochemical impedance approach, Chem. Commun. Chem. Commun. 52 (2016) 11406-11409. doi:10.1039/c6cc06006d. 
[29] M. Lindgren, J. Perolainen, Slurry pot investigation of the influence of erodent characteristics on the erosion resistance of austenitic and duplex stainless steel grades, Wear. 319 (2014) 38-48. doi:10.1016/j.wear.2014.07.006.

[30] J. Malik, I.H. Toor, W.H. Ahmed, Z.M. Gasem, M.A. Habib, R. Ben-Mansour, H.M. Badr, Evaluating the effect of hardness on erosion characteristics of aluminum and steels, J. Mater. Eng. Perform. 23 (2014) 2274-2282. doi:10.1007/s11665-014-1004-X.

[31] M.A. Nemitallah, R. Ben-Mansour, M. a. Habib, W.H. Ahmed, I.H. Toor, Z.M. Gasem, H.M. Badr, Solid Particle Erosion Downstream of an Orifice, J. Fluids Eng. 137 (2014) 021302. doi:10.1115/1.4028283.

[32] S.S. Rajahram, T.J. Harvey, R.J.K. Wood, Electrochemical investigation of erosioncorrosion using a slurry pot erosion tester, Tribol. Int. 44 (2011) 232-240. doi:10.1016/j.triboint.2010.10.008.

[33] J.I. Ukpai, R. Barker, X. Hu, A. Neville, Determination of particle impacts and impact energy in the erosion of X65 carbon steel using acoustic emission technique, Tribol. Int. 65 (2013) 161-170. doi:10.1016/j.triboint.2013.03.012.

[34] Z.B. Zheng, Y.G. Zheng, X. Zhou, S.Y. He, W.H. Sun, J.Q. Wang, Determination of the critical flow velocities for erosion-corrosion of passive materials under impingement by $\mathrm{NaCl}$ solution containing sand, Corros. Sci. $88 \quad$ (2014) 187-196. doi:10.1016/j.corsci.2014.07.043.

[35] J. Fransaer, J.P. Celis, J.R. Roos, Variations in the flow of current to disk electrodes caused by particles, J. Electroanal. Chem. 391 (1995) 11-28. doi:10.1016/00220728(94)03855-W. 
[36] J. Fransaer, V. Bouet, J. Celis, C. Gabrielli, E. Huet, G. Maurin, Perturbation of the Flow of Current to a Disk Electrode by an Insulating Sphere, J. Electrochem. Soc. 142 (1995) $4181-4189$.

[37] H.S. Grewal, H. Singh, E.S. Yoon, Interplay between erodent concentration and impingement angle for erosion in dilute water-sand flows, Wear. 332-333 (2015) 11111119. doi:10.1016/j.wear.2015.02.039.

[38] P.R. Birkin, D.G. Offin, T.G. Leighton, The study of surface processes under electrochemical control in the presence of inertial cavitation, Wear. 258 (2005) 623628. doi:10.1016/j.wear.2004.05.025.

[39] K. Sasaki, G.T. Burstein, Observation of a threshold impact energy required to cause passive film rupture during slurry erosion of stainless steel, Philos. Mag. Lett. 80 (2000) 489-493. doi:10.1080/09500830050057198.

[40] G.T. Burstein, A.J. Davenport, The Current-Time Relationship during Anodic Oxide Film Growth under High Electric Field, J. Electrochem. Soc. 136 (1989) 936-941.

[41] G.T. Burstein, D.H. Davies, The Electrochemical behaviour of scratched iron surfaces in aqueous solutions, J. Electrochem. Soc. 128 (1981) 33-39.

[42] D.C. Giancoli, Physics for Scientists and Engineers with Modern Physics, 2nd ed., Prentice-Hall, New Jersey, 1988. 